\title{
In-band pumping avenue based high power superfluorescent fiber source with record power and near-diffraction-limited beam quality
}

\author{
Jiangming $\mathrm{Xu}^{1,2}$, Jun $\mathrm{Ye}^{1}$, Hu Xiao ${ }^{1,2}$, Jinyong Leng ${ }^{1,2}$, Wei Liu ${ }^{1}$, and Pu Zhou ${ }^{1,2}$ \\ ${ }^{1}$ College of Optoelectronic Science and Engineering, National University of Defense Technology, Changsha 410073, China \\ ${ }^{2}$ Hunan Provincial Collaborative Innovation Center of High Power Fiber Laser, Changsha 410073, China \\ (Received 26 April 2018; revised 9 July 2018; accepted 16 July 2018)
}

\begin{abstract}
High power superfluorescent fiber sources (SFSs), which could find wide applications in many fields such as middle infrared laser generation, Raman fiber laser pumping and spectral beam combination, have experienced a flourishing time in recent years for its unique properties, such as short coherence length and high temporal stability. The challenge for performance scalability of powerful SFS mainly lies on the physical issues including parasitic laser oscillation and modal instability (MI). In this contribution, by employing in-band pumping avenue and high-order transverse-mode management, we explore a high power SFS with record power, near-diffraction-limited beam quality and spectral manipulation flexibility. An ultimate output power of $3.14 \mathrm{~kW}$ can be obtained with high temporal stability and a beam quality of $M^{2}=1.59$ for the amplified light. Furthermore, the dynamics of spectral evolutions, including redshifting of central wavelength and unsymmetrical broadening in spectral wings, of the main amplifier with different seed linewidths are investigated contrastively. Benefiting from the unique high pump brightness and high MI threshold of in-band pumping scheme, the demonstrated system also manifests promising performance scaling potential.
\end{abstract}

Keywords: fiber amplifier; high power; in-band pumping; superfluorescent fiber source

\section{Introduction}

Thanks to the superior features on short coherence length, high temporal and thermal stability, broad spectral coverage and good beam quality, superfluorescent fiber sources (SFSs) have drawn great attention in the past decades ${ }^{[1-4]}$, and researches, including spectral evolution ${ }^{[5]}$, operating wavelength expansion ${ }^{[6,7]}$, and potential application exploration ${ }^{[8,9]}$ have been widely investigated. Especially, the utilizations of high power SFSs in many fields, such as mid-infrared laser and supercontinuum generation ${ }^{[10,11]}$, random fiber laser, and Raman fiber laser pumping ${ }^{[12-14]}$ and spectral beam combination ${ }^{[15]}$, have been demonstrated in recent years for its inherent unique incoherence and high temporal stability characteristics. Consequently, the performance scalability of high power SFS is extremely significant.

As early as 2006, Wang et al. demonstrated the first hundred-watt-level SFS in a single-stage structure operating

Correspondence to: P. Zhou, College of Optoelectronic Science and Engineering, National University of Defense Technology, No. 109 Deya Road, Changsha 410073, China. Email: zhoupu203@163.com in the $1 \mu \mathrm{m}$ region by employing multi-mode-offset-core fiber to suppress the parasitic lasing ${ }^{[16]}$. The highest powers ever reported from single-stage structured SFSs are $186.3 \mathrm{~W}^{[17]}, 16 \mathrm{~W}^{[18]}$ and $46 \mathrm{~W}^{[19]}$ for $1 \mu \mathrm{m}, 1.5 \mu \mathrm{m}$, and $2 \mu \mathrm{m}$ range, respectively. Although previous investigations have announced that hundred-watt-level high power SFS can be achieved in a single-stage structure with careful system parameters design, the further power enhancement is still a challenge because of the physical parasitic lasing issue. What is more, the presented high power SFSs in single-stage structure were often broadband and randomlypolarized $^{[16-19]}$, while narrowband and polarization characteristics are required in practical applications ${ }^{[20,21]}$. Fortunately, employing master-oscillator-power-amplifier (MOPA) configuration can introduce a new horizon of high power SFS and leverage the high power SFS with special spectrum/polarization characteristics ${ }^{[22-25]}$ and kilowatts operating power ${ }^{[26-30]}$. Besides, the output light of MOPAstructured SFS, in contrast to that of fiber MOPA seeded by standard resonant-cavity-based oscillator with self-pulsing effect, also has the advantages of high temporal stability ${ }^{[2,4]}$. Despite a maximal power of $2.53 \mathrm{~kW}$ achieved from 
MOPA-structured SFS ${ }^{[30]}$, the generation of thermal effect induced modal instability (MI), which is often associated with beam quality deteriorating ${ }^{[31,32]}$, can be measured at only about $2 \mathrm{~kW}$ output power ${ }^{[28,30]}$. As the investigations indicated, in-band pumping avenue, which can increase pump brightness and decrease quantum loss simultaneously, has the potential to decrease the thermal load in the gain fiber and increase the MI generation threshold ${ }^{[33-36]}$. Thus, in our previous works, we have also pointed out theoretically that in-band pumping route can boost the MI threshold ${ }^{[37]}$ and verified that in-band pumping avenue could be applied for the power advancing of SFS with $1.31 \mathrm{~kW}$ maximum output power $^{[4]}$. Here, we explore the operating power of SFS to a record level $(3.14 \mathrm{~kW})$ with excellent beam quality, spectral manipulation flexibility and great power scaling potential features by employing in-band pumping avenue and effective high-order transverse-mode management.

\section{Experimental setup}

A schematic of the high power MOPA-structured SFS system is plotted in Figure 1. The initial seed is a broadband amplified spontaneous emission (ASE) source and the pump light is provided by a fiber pigtailed laser diode (LD) centered at $976 \mathrm{~nm}$ with maximal output power of about $9 \mathrm{~W}$. The pump light is injected into the gain fiber via a $(2+1) \times$ 1 signal/pump combiner. The pump delivery fibers of the $\mathrm{LD}$ and the combiner are both $105 / 125 \mu \mathrm{m}$ with numerical aperture (NA) of 0.22 . A piece of $5 \mathrm{~m}$ long dual-cladding ytterbium doped fiber (YDF) with $10 / 125 \mu \mathrm{m}$ core/inner cladding diameters is employed as the gain fiber. The average cladding absorption coefficient for $976 \mathrm{~nm}$ pump light is about $6 \mathrm{~dB} / \mathrm{m}$. The end of the YDF is angle-cleaved $\left(8^{\circ}\right)$ to decrease the reflection and suppress parasitic oscillation. To protect the seed source from potential backward light, a broadband fiber isolator (ISO) operating at $1080 \pm 30 \mathrm{~nm}$ is spliced after the signal port of the combiner, and the fusing region is covered with high-index gel to form a cladding light stripper (CLS). To select a small piece from the broadband seed light, a flexible spectral filter module composed by a fiber circulator and a narrowband fiber Bragg grating (FBG) is employed after the broadband seed. The FBG (reflectivity $\sim 99 \%$ ) centered at $1080 \mathrm{~nm}$ with full width at half maximum (FWHM) of about $2 \mathrm{~nm}$ is spliced to the $2 \mathrm{nd}$ port of the circulator, which acts as a spectral selection mirror. Then, the filtered narrowband light can be obtained from the 3rd port of the circulator.

A two-stage pre-amplifier is utilized to scale the seed light to a suitable power level. Additionally, a high power fiber circulator ( $30 \mathrm{~W}$ maximal operating power) is inserted between the pre-amplifiers to monitor the backward light. In the main amplifier stage, an in-band pumping strategy is utilized. Twenty four channels of homemade $1018 \mathrm{~nm}$ fiber

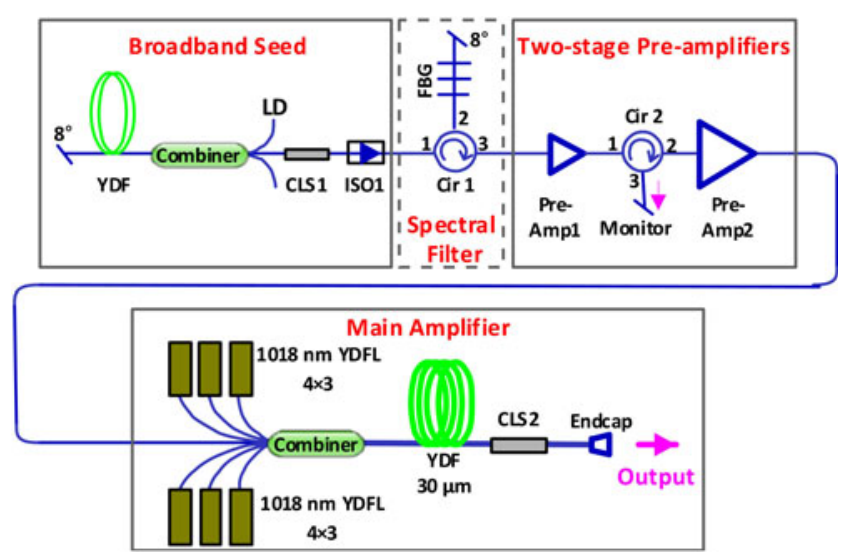

Figure 1. A schematic of the in-band pumped high power SFS system. YDF, Yb-doped fiber; LD, laser diode; CLS, cladding light stripper; ISO, isolator; FBG, fiber Bragg grating; Cir, circulator; Pre-Amp, pre-amplifier; YDFL, Yb-doped fiber laser.

laser are employed as the pump light with $150-180 \mathrm{~W}$ output power per channel (see Ref. [38] and related literature). The pump lasers are first combined into six groups with four pump combiners $(4 \times 1$, homemade; see Ref. [39] and related literature). The input and output fibers of the $4 \times 1$ pump combiner exhibit $15 \mu \mathrm{m}(0.08 \mathrm{NA})$ and $105 \mu \mathrm{m}(0.22 \mathrm{NA})$ core diameters, respectively. Then, the six groups of pump lasers and pre-amplified seed light are launched into the main amplifier via a commercial $(6+1) \times 1$ pump/signal combiner (provided by Lightcomm. Technology Co., Ltd.), whose input and output signal ports exhibit $10 \mu \mathrm{m}$ and $30 \mu \mathrm{m}$ core diameters, respectively. A piece of $30 \mathrm{~m}$ YDF (fabricated by China Electronics Technology Corporation) exhibiting $30 \mu \mathrm{m}$ core diameter and average cladding absorption coefficient of $0.45 \mathrm{~dB} / \mathrm{m}$ for $1018 \mathrm{~nm}$ pump laser is utilized as a gain fiber in the main amplification stage. For the thermal dissipation, the YDF is coiled on the surface of a watercooling aluminum plate with a diameter of about $15 \mathrm{~cm}$. Then, an endcap is employed to deliver the amplified SFS into free space. And the fusing region of the gain fiber and the pigtailed fiber of the endcap is covered with high-index gel to strip the residual pump and leaky signal light in the inner cladding.

\section{Results and discussion}

For the broadband seed source without spectral filtering, the ultimate output power after fiber isolator (ISO1) is $1.3 \mathrm{~W}$. Figure 2 depicts the spectral characteristics at the maximum power level. The corresponding central wavelength and FWHM linewidth are $1079.4 \mathrm{~nm}$ and $10.3 \mathrm{~nm}$, respectively. With the aid of the spectral filter, narrowband seed can be obtained with FWHM linewidth of $1.8 \mathrm{~nm}$ and maximal power of $84.5 \mathrm{~mW}$. Then, the narrowband seed light is boosted to $104.4 \mathrm{~W}$ by the two-stage preamplifiers. The spectral characteristics can maintain well 


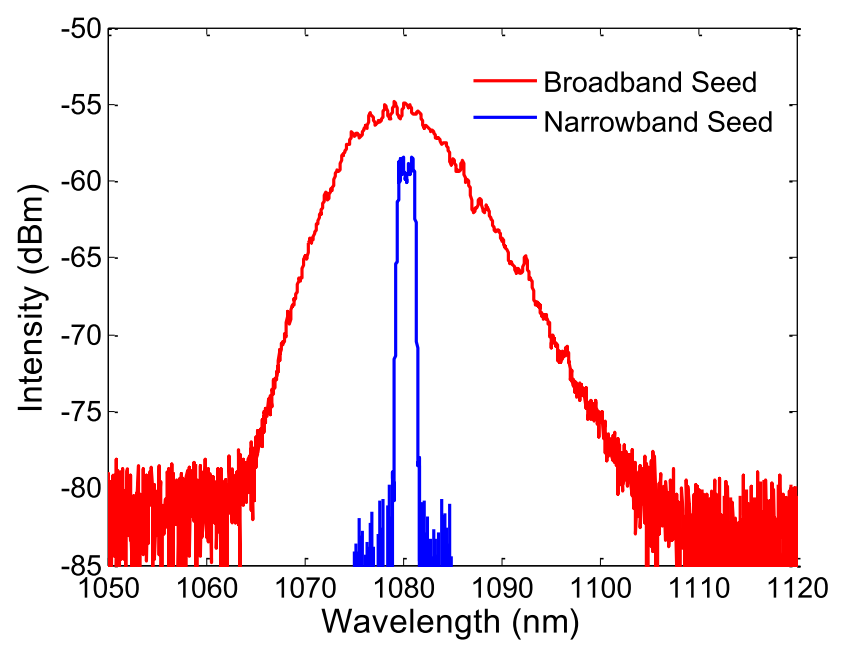

Figure 2. Spectral characteristics of the seed source.

in the pre-amplification process. Additionally, benefiting from the replaceable feature of the spectral filtering element, the operable wavelength and linewidth of demonstrated SFS seed can be manipulated conveniently ${ }^{[40]}$.

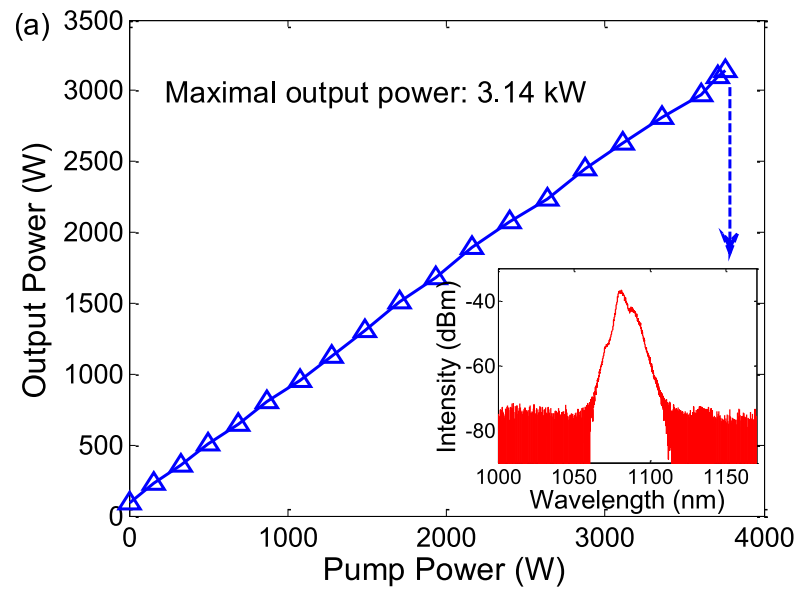

The output characteristics of the main amplifier are shown in Figure 3. The ultimate output power is $3.14 \mathrm{~kW}$ for given $3.76 \mathrm{~kW}$ pump light, corresponding to an opticalto-optical conversion efficiency of $80.74 \%$. As the total absorption coefficient of the gain fiber for $1018 \mathrm{~nm}$ pump laser is about $13.5 \mathrm{~dB}$ (about 95.54\%), the opticalto-optical conversion efficiency is about $84.51 \%$ with the consideration of unabsorbed pump laser. However, considering the $71 \%$ optical-to-optical conversion efficiency of the $1018 \mathrm{~nm}$ pumping laser ${ }^{[38]}$, the overall efficiency of the main amplification stage is about $60.00 \%$. The output spectrum at maximum power level is depicted in the inset of Figure 3(a). Most of the pump laser is absorbed or stripped, and the stimulated Raman scattering component can be neglected with an amplitude of at least $36 \mathrm{~dB}$ lower than the main signal peak. Furthermore, the FWHM linewidth broadens to about $4.68 \mathrm{~nm}$, which is different from the previously reported narrowband MOPA-structured spectralbroaden-free SFS in Ref. [29] pumped by the LD. As the linewidth broadening is mainly induced by the generation of nonlinear effects ${ }^{[41]}$, such as self-phase modulation (SPM) and cross-phase modulation (XPM), the difference may be

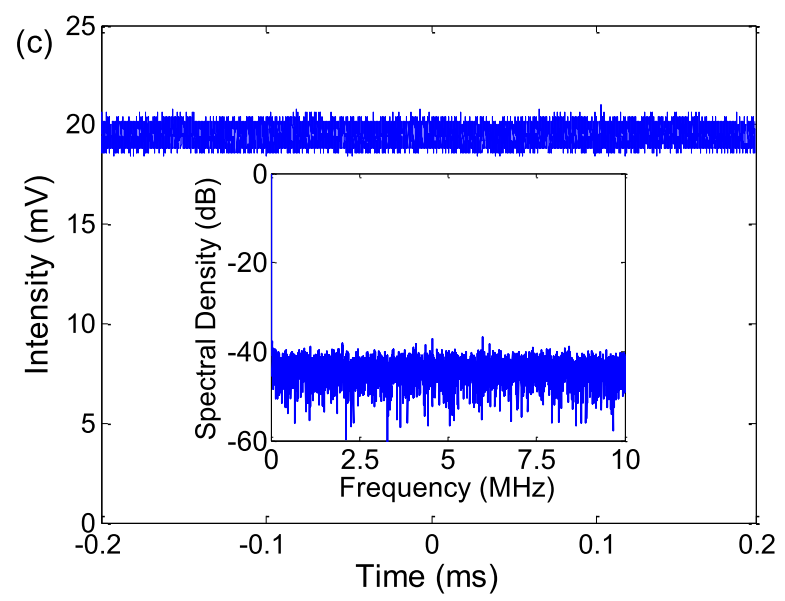

Figure 3. Output characteristics of the main amplifier. (a) Output power as a function of pump power and spectrum at the ultimate power. (b) Measured beam quality at the maximal power. (c) Characteristics in time and frequency domains at full power. 

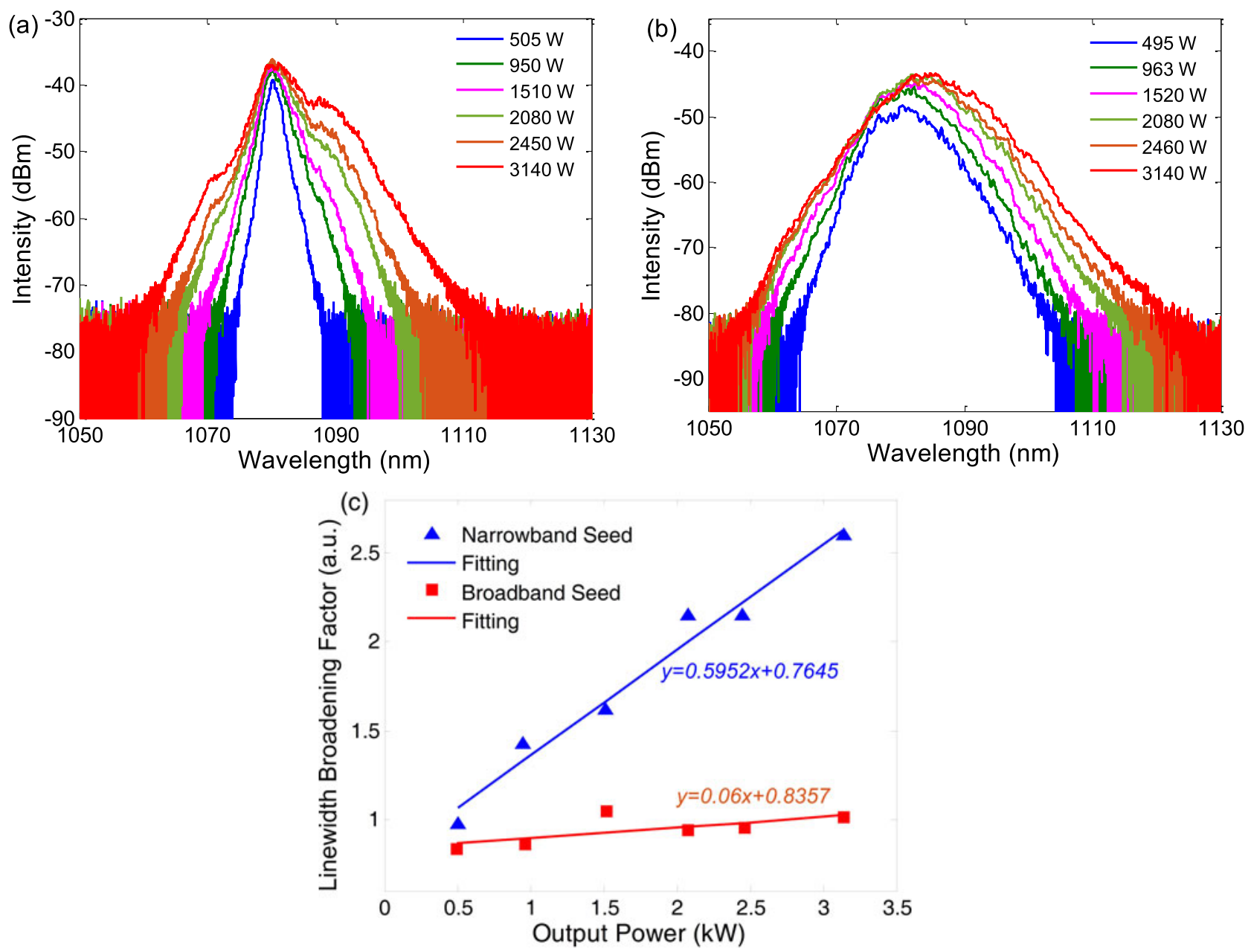

Figure 4. Spectral characteristics of amplified light. Spectral details of (a) narrowband seed amplification and (b) broadband seed amplification. (c) FWHM linewidth broadening factors as functions of output power.

induced by the relative long gain fiber employed in this scheme. The spectral evolution in the power scaling process will be investigated in detail in the following paragraph.

The beam quality of the output light maintains well in the power scaling process, and a beam quality of $M^{2}=1.59$ is measured at the maximal power, as shown in Figure 3(b). The beam quality measurement indicates that no MI is generated in the power scaling process. We want to stress that nearly single transverse-mode-amplified light can be acquired via effective high-order transverse-mode management despite the employment of non-strict-single-mode YDF in the main amplification stage. In the power amplification, the coil diameter is optimized to be about $15 \mathrm{~cm}^{[42-44]}$. Then total bending loss of about $18 \mathrm{~dB}$ can be obtained for the $\mathrm{LP}_{11}$ mode in the $30 \mathrm{~m}$ long YDF, while the bending loss for $\mathrm{LP}_{01}$ mode can be neglected. Based on this effective high-order transverse-mode management, near-diffractionlimited output beam can be obtained. An InGaAs photo detector ( $5 \mathrm{GHz}$ bandwidth, rise time $<70 \mathrm{ps}$ ) and a digital phosphor oscilloscope (1 GHz bandwidth, $5 \mathrm{GS} / \mathrm{s}$ sampling rate) are utilized to measure the temporal characteristic. The corresponding standard deviation (STD) of the measured temporal signal is calculated to be about $1.86 \%$. Further spectral density analysis of the temporal signal at full power, as depicted in the inset of Figure 3(c), exhibits that no characteristic frequency component can be measured and demonstrates that no parasitic oscillation or self-pulsation is generated in the power scaling process.

For comparison, broadband seed without spectral filtration is also amplified by removing the spectral filter from the MOPA system to investigate the influence of seed linewidth on the output spectrum in the power amplification process. The same maximum output power of $3.14 \mathrm{~kW}$ broadband SFS can be obtained. No obvious difference can be found in the output power and beam quality characteristics. However, the spectral evolutions in the power enhancing process exhibit evident difference. The spectral details of amplified light at different power levels from the MOPA system with narrowband seed and broadband seed are plotted in 
Figures 4(a) and 4(b), respectively. As to the narrowband seed amplification, the gain magnification of the long wavelength section (around $1090 \mathrm{~nm}$ ) is superior to that of the short wavelength section (around $1070 \mathrm{~nm}$ ) during the power scaling to $2080 \mathrm{~W}$, which might be attributed to the influence of factors such as the operating temperature and the gain cross-sections of the gain fiber. With further power scaling, the short wavelength section around $1070 \mathrm{~nm}$ will be enhanced simultaneously for the nonlinear effect of four wave mixing (FWM) between the wavelength compositions around $1080 \mathrm{~nm}$ and $1090 \mathrm{~nm}$. Similarly, the long wavelength section also exhibits the superiority in broadband amplification. Unlikely, the central wavelength of amplified light red-shifts drastically to $1084.56 \mathrm{~nm}$.

Since the linewidth property is important for many applications, the linewidth evolutions are investigated with the consideration of the linewidth broadening factor, which is defined as the ratio of the FWHM linewidth of the amplified light to the injected seed. The linear fitting result shown in Figure 4(c) indicates that the FWHM linewidth broadening factor of narrowband seed amplification increases with a rate of about $0.5952 \mathrm{~nm}$ per kilowatt, which is about 10 times as large as that of broadband seed amplification. This may be induced by the differences of the spectrum density and intensity noise of the seeds ${ }^{[41,45,46]}$. As the linewidth broadening in the narrowband seed amplification is relatively more serious than that in the broadband seed amplification, the optimization of the FWHM linewidth of the employed seed is very important for the design of the powerful SFS system if special output linewidth is required. Additionally, the quantitative analysis and effective manipulation of spectral broadening phenomenon based on a more comprehensive theoretical model is our next goal.

\section{Conclusion}

In conclusion, we present an in-band pumping avenue enabled high power SFS with a record output power. Based on the homemade 24 channels of $1018 \mathrm{~nm}$ fiber laser, the in-band pumping scheme has been employed to avoid the generation of MI and enhance the brightness of pump laser. The ultimate output power of the main amplifier is $3.14 \mathrm{~kW}$, corresponding to an optical-to-optical conversion efficiency of $80.74 \%$. A beam quality of $M^{2}=1.59$ is measured at the maximal power. The beam quality and temporal stability measurements indicate that no MI was generated even at the ultimate operation power. Furthermore, the influence of FWHM linewidth of the seed on the amplified light is investigated contrastively. The output power is limited only by the pump power, and further power scaling of this MOPA-structured SFS is available. Moreover, benefiting from the flexible feature of the spectral filtering element, the wavelength and linewidth manipulations can also be realized conveniently.

\section{Acknowledgements}

This work was supported in part by the National Natural Science Foundation of China (No. 61322505), the Foundation for the author of National Excellent Doctoral Dissertation of China (No. 201329), the Huo Yingdong Education Foundation of China (No. 151062), and the Hunan Provincial Innovation Foundation for Postgraduate Student (No. CX2017B030). The authors also would like to thank Dr. Xiaoming Xi for revising this manuscript.

\section{References}

1. S. Martin-Lopez, M. Gonzalez-Herraez, A. Carrasco-Sanz, F. Vanholsbeeck, S. Coen, H. Fernandez, J. Solis, P. Corredera, and M. L. Hernanz, Meas. Sci. Technol. 17, 1014 (2006).

2. P. Wang, J. K. Sahu, and W. A. Clarkson, IEEE J. Sel. Top. Quant. Electron. 13, 580 (2007).

3. H. Zhang, X. Shen, H. Hao, Q. Li, and M. Gong, High Power Laser Sci. Eng. 4, e31 (2016).

4. J. Xu, P. Zhou, W. Liu, J. Leng, H. Xiao, P. Ma, J. Wu, H. Zhang, J. Chen, and Z. Liu, IEEE J. Sel. Top. Quant. Electron. 24, 0900710 (2018).

5. P. Yan, J. Sun, D. Li, M. Gong, and Q. Xiao, Opt. Commun. 380, 250 (2016).

6. K. E. Riumkin, M. A. Mel'kumov, I. A. Bufetov, A. V. Shubin, S. V. Firstov, V. F. Khopin, A. N. Gur'yanov, and E. M. Dianov, Opt. Lett. 37, 4817 (2012).

7. H. Luo, J. Li, L. Wang, Y. He, H. Li, and Y. Liu, IEEE Photon. Technol. Lett. 26, 2287 (2014).

8. B. Redding, P. Ahmadi, V. Mokan, M. Seifert, M. A. Choma, and H. Cao, Opt. Lett. 40, 4607 (2015).

9. H. Maestre, A. J. Torregrosa, and J. Capmany, Opt. Express 24, 8581 (2016).

10. Y. Shang, J. Xu, P. Wang, X. Li, P. Zhou, and X. Xu, Opt. Express 24, 21684 (2016).

11. A. Jin, H. Zhou, X. Zhou, J. Hou, and Z. Jiang, IEEE Photon. J. 7, 1600409 (2015).

12. J. Xu, Z. Lou, J. Ye, J. Wu, J. Leng, H. Xiao, H. Zhang, and P. Zhou, Opt. Express 25, 5609 (2017).

13. J. Xu, J. Ye, W. Liu, J. Wu, H. Zhang, J. Leng, and P. Zhou, Photon. Res. 5, 598 (2017).

14. W. Pan, L. Zhang, J. Zhou, X. Yang, and Y. Feng, Opt. Lett. 42, 5162 (2017)

15. Y. Zheng, Y. Yang, J. Wang, M. Hu, G. Liu, X. Zhao, X. Chen, K. Liu, C. Zhao, B. He, and J. Zhou, Opt. Express 24, 12063 (2016).

16. P. Wang, J. K. Sahu, and W. A. Clarkson, Opt. Lett. 31, 3116 (2006).

17. Y. An, Y. Yu, J. Cao, Z. Huang, S. Guo, and J. Chen, Laser Phys. Lett. 13, 025105 (2016).

18. W. Chen, D. Shen, T. Zhao, and X. Yang, Opt. Express 20, 14542 (2012).

19. Z. Hu, P. Yan, Q. Liu, E. Ji, Q. Xiao, and M. Gong, Appl. Phys. B 118, 101 (2015).

20. M. Jiang, P. Ma, L. Huang, J. Xu, P. Zhou, and X. Gu, High Power Laser Sci. Eng. 5, e30 (2017).

21. P. Zhou, L. Huang, J. Xu, P. Ma, R. Su, J. Wu, and Z. Liu, Sci. China Tech. Sci. 60, 1784 (2017).

22. O. Schmidt, M. Rekas, C. Wirth, J. Rothhardt, S. Rhein, A. Kliner, M. Strecker, T. Schreiber, J. Limpert, R. Eberhardt, and A. Tünnermann, Opt. Express 19, 4421 (2011). 
23. P. Yan, J. Sun, D. Li, X. Wang, Y. Huang, M. Gong, and Q. Xiao, Opt. Express 24, 19940 (2016).

24. P. Wang and W. A. Clarkson, Opt. Lett. 32, 2605 (2007).

25. P. Ma, R. Tao, X. Wang, P. Zhou, and Z. Liu, IEEE Photon. Technol. Lett. 27, 879 (2015).

26. O. Schmidt, A. Kliner, M. Rekas, C. Wirth, S. Rhein, T. Schreiber, R. Eberhardt, and A. Tuennermann, in Proc. Front. Opt. (OSA, 2011), paper FTuW3.

27. J. Xu, L. Huang, J. Leng, H. Xiao, S. Guo, P. Zhou, and J. Chen, Opt. Express 23, 5485 (2015).

28. Y. Qi, M. Lei, C. Liu, B. He, and J. Zhou, in Proc. CLEO: Appl. Technol. (OSA, 2015), paper ATu4M-4.

29. J. Xu, W. Liu, J. Leng, H. Xiao, S. Guo, P. Zhou, and J. Chen, Opt. Lett. 40, 2973 (2015).

30. J. Xu, H. Xiao, J. Leng, H. Zhang, P. Zhou, and J. Chen, Laser Phys. Lett. 13, 105101 (2016).

31. T. Eidam, C. Wirth, C. Jauregui, F. Stutzki, F. Jansen, H. J. Otto, O. Schmidt, T. Schreiber, J. Limpert, and A. Tünnermann, Opt. Express 19, 13218 (2011).

32. C. Jauregui, J. Limpert, and A. Tünnermann, Nat. Photon. 7, 861 (2013).

33. M. A. Jebali, J. N. Maran, and S. Larochelle, Opt. Lett. 39, 3974 (2014).

34. M. N. Zervas and C. A. Codemard, IEEE J. Sel. Top. Quant. Electron. 20, 219 (2014).
35. P. Zhou, H. Xiao, J. Leng, J. Xu, Z. Chen, H. Zhang, and Z. Liu, J. Opt. Soc. Am. B 34, A29 (2017).

36. X. Jin, Z. Lou, Y. Chen, P. Zhou, H. Zhang, H. Xiao, and Z. Liu, Sci. Rep. 7, 42402 (2017).

37. R. Tao, P. Ma, X. Wang, P. Zhou, and Z. Liu, IEEE J. Quant. Electron. 51, 1600106 (2015).

38. H. Xiao, P. Zhou, X. Wang, X. Xu, and Z. Liu, Laser Phys. Lett. 10, 065102 (2013)

39. H. Zhou, A. Jin, Z. Chen, B. Zhang, X. Zhou, S. Chen, J. Hou, and J. Chen, Opt. Lett. 40, 3810 (2015).

40. X. Wang, X. Jin, P. Zhou, X. Wang, H. Xiao, and Z. Liu, Opt. Express 23, 3382 (2015).

41. W. Liu, P. Ma, H. Lv, J. Xu, P. Zhou, and Z. Jiang, Opt. Express 24, 8708 (2016).

42. L. Huang, L. Kong, J. Leng, P. Zhou, S. Guo, and X. Cheng, J. Opt. Soc. Am. B 33, 1030 (2016).

43. R. Tao, R. Su, P. Ma, X. Wang, and P. Zhou, Laser Phys. Lett. 14, 025101 (2017).

44. L. Kong, J. Leng, P. Zhou, and Z. Jiang, Opt. Express 25, 23437 (2017)

45. L. Huang, J. Xu, J. Ye, X. Liu, H. Zhang, X. Wang, and P. Zhou, IEEE J. Sel. Top. Quant. Electron. 24, 0900608 (2018).

46. S. I. Kablukov, E. A. Zlobina, E. V. Podivilov, and S. A. Babin, Opt. Lett. 37, 2508 (2012). 